\title{
A Case of Sarcoidosis Disseminated to Skeletal Tissues
}

Edon Rabinowitz', Chinwe Ogedegbe², Joseph Feldman².

\begin{abstract}
.
Sarcoidosis is a systemic granulomatous disease of unknown etiology that typically affects young adults. Diagnostic criteria for sarcoidosis include involvement of two or more of the following organ systems: 1) pulmonary infiltrates; 2) bilateral hilar adenopathy; and 3) skin and/or eye lesions. Musculoskeletal system is less commonly involved. For that reason potential presenting symptoms can vary and make the diagnosis very challenging; particularly if a patient has symptoms that mimic other conditions. Musculoskeletal involvement for example can mimic malignancy. The following case describes a patient with known history of primary metastatic mediastinal Germ Cell Tumor (CCT) with teratomatous elements who is diagnosed with sarcoidosis involving skeletal tissues.
\end{abstract}

Keywords: Sarcoidosis; Musculoskeletal System; Neoplasms, Germ Cell and Embryonal (Source: MeSH, NLM).

\section{Introduction.}

Sarcoidosis is a chronic inflammatory disease, characterized by noncaseating granulomas, which can affect any organ, but most commonly the lungs. The diagnosis of sarcoidosis is one of exclusion after ruling out pathologies such as mycobacterial, fungal infections, malignancy etc. The etiology of Sarcoidosis is unknown but is theorized to be an infectious or noninfectious environmental agent that triggers an inflammatory response in a genetically susceptible host. Clinical features include nonproductive cough and shortness of breath on exertion. Hallmark findings include bilateral hilar adenopathy on chest $\mathrm{x}$-ray and elevated serum Angiotensin-converting enzyme and calcium. Systemic findings include erythema nodosum, uveitis, arthralgias and arthritis. Sarcoidosis is typically managed with systemic corticosteroids.

Cases of Sarcoidosis are annually documented worldwide at varying incidence rates. Diagnosis most often occurs under the age of 50-years-old, peaking in the 3 rd and 4 th decades of life $^{2}$ with the highest incidence (5-40 cases per 100,000) reported in Northern European nations, particularly Ireland and Scandinavia. ${ }^{3}$ In the United Sates, African Americans are approximately three times more likely to be affected by sarcoidosis compared to Caucasians ( 35.5 cases per 100,000 versus to 10.9 cases per 100,000 respectively). ${ }^{4}$ Despite geographic and racial differences, sarcoidosis uniformly shows a greater preponderance towards females. ${ }^{5}$

It is well documented that over $90 \%$ of cases of Sarcoidosis have pulmonary, cutaneous or ocular involvement with little of no skeletal involvement. ${ }^{6}$ Even though detection of osseous involvement in Sarcoidosis has been increasing due to improved imaging techniques such as MRI and PET, true incidence rate have been difficult to assess for several reasons. First is the asymptomatic nature of osseous involvement. Secondly, because when symptoms of bone pain do exist, their diagnosis is obscured by classic musculoskeletal clinical manifestations of Sarcoidosis including arthralgias, sarcoid arthritis and sarcoid myopathies. Thirdly, as seen in the case to be described below, because skeletal involvement in Sarcoidosis can be misinterpreted as metastatic involvement of the bones. Radiographically reported incidence of skeletal involvement in the setting of Sarcoidosis occurs in only $5 \%$ of patients. ${ }^{7}$

\section{The Case.}

A 33-year-old Caucasian male, with past medical history significant for mediastinal Germ Cell Tumor (CCT), presented to the emergency department (ED) with 2-month history of worsening exertional dyspnea. When diagnosed with GCT 3 years ago, CAT scan imaging of his chest showed pathologically enlarged lymph nodes extending from the anterior mediastinum into the middle mediastinum measuring 8.1 $x 10.0 \mathrm{~cm}$ on the right and $8.0 \times 9.6 \mathrm{~cm}$ on the left. Subsequent lymph node biopsy revealed immature GCT consisting predominantly of yolk sac tumor with teratomatous component. Testicular ultrasound was unremarkable at that time.

The patient's dyspnea significantly worsened over the week prior. He reported a productive cough with clear sputum and denied palpitations or chest pain. On physical exam
About the Author Dr. Edon Rabinowitz is a graduate of Binghamton University in New York, and St. George's University School of Medicine, Cum Laude, recipient of the Legacy of Excellence Scholarship. He is currently a first year pedia tric resident at Steven and Alexandra Cohen Children's Medical Center at Hofstra North Shore LIJ School of Medicine, Long Island, NY.

Submission: 2011, Dec 6 Accepted: 2012, March 26. Process: Peer-Reviewed.

\footnotetext{
${ }^{1}$ St George's University, Saint George's, Grenada, West Indies.

${ }^{2}$ Hackensack University Medical Center, Hackensack, New Jersey.

Correspondence

Edon Rabinowitz

Address: St George's University, Saint George's, Grenada, West Indies.

E. mail: rabedo@sgu.edu
} 
he was afebrile, tachycardic, and tachypneic and found to be in moderate respiratory distress. Bibasilar crepitus was noted and arterial blood gas revealed a $\mathrm{PaO}_{2}$ of $57.3 \mathrm{mmHg}$ on room air, indicating hypoxemia. A follow up CAT scan revealed a $14.4 \times 11.3 \mathrm{~cm}$ anterior mediastinal mass with diffuse reticulonodular infiltrates in both lungs. Subsequent left lung video-assisted thoracoscopy and biopsy found

Figure 1. Abnormal hypermetabolism of the left trochanteric region. These PET/CT images were interpreted by evaluation of both the anatomic (CT) and metabolic (PET) data which were electronically fused.
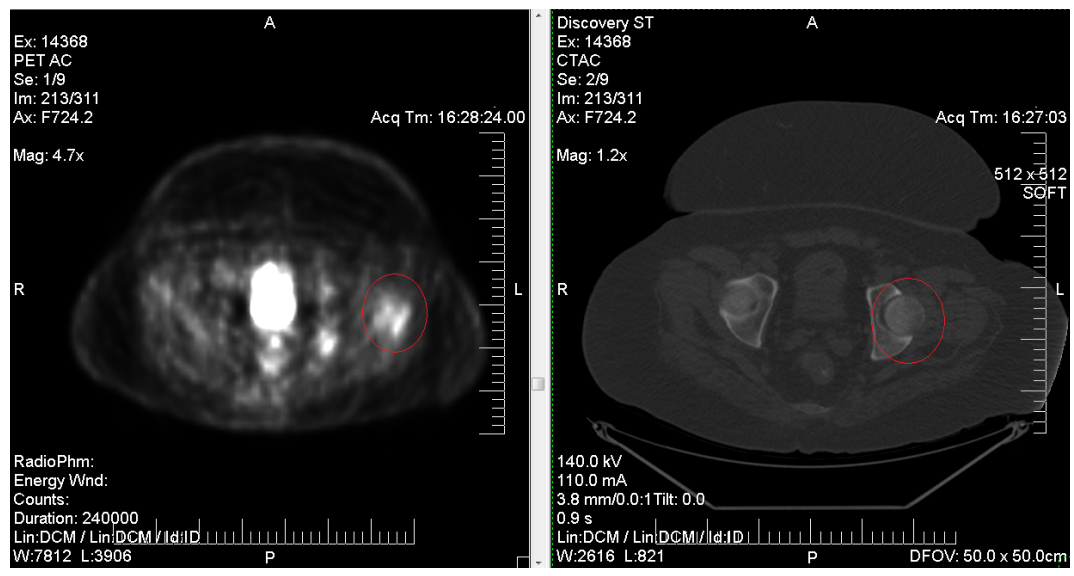

Figure 2. Abnormal hypermetabolism corresponding to $L 3$ vertebra.
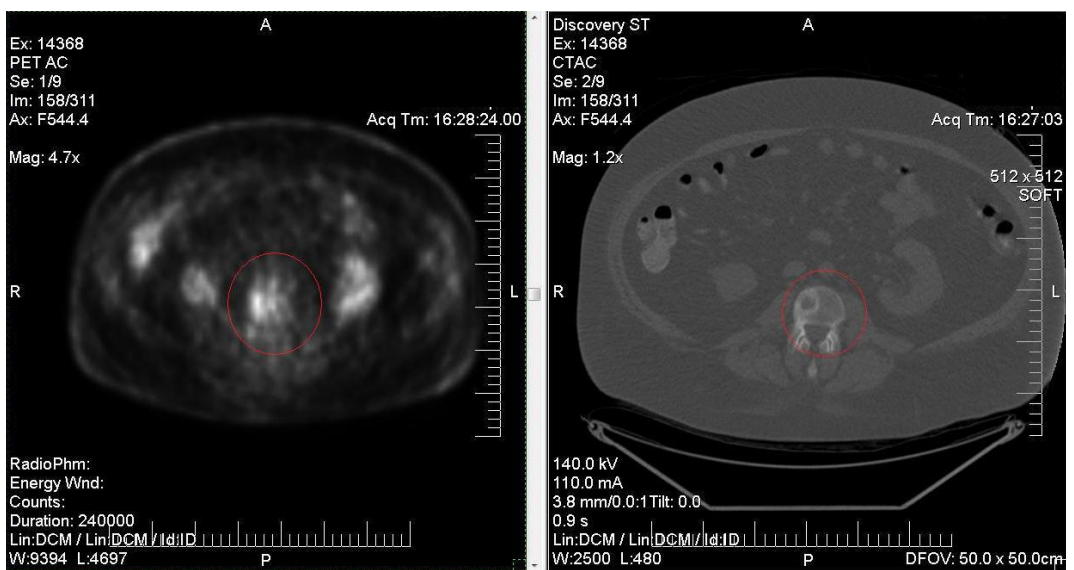

Figure 3. Mild abnormal hypermetabolism in fusiform fashion of the right third lateral rib. Additional nonosseous regions of hypermetabolism are seen corresponding to pathologically enlarged mediastinal and hilar lymph nodes.
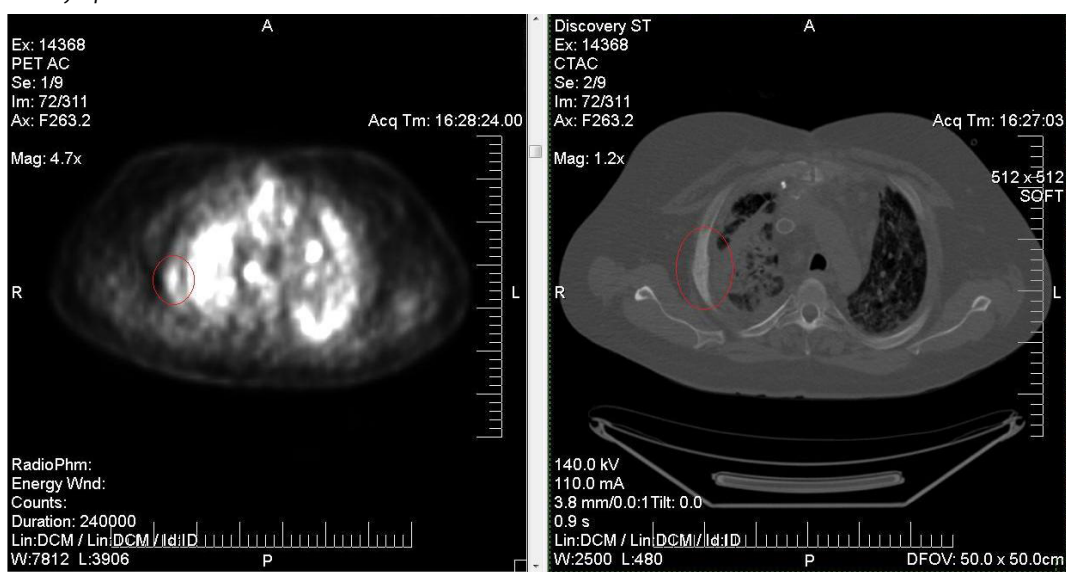

predominantly well-defined large multinucleated giant cell granulomas consistent with Sarcoidosis. The patient was started on oral steroid therapy without improvement in his symptoms.

Over the ensuing 6 months the disease progressed and he presented to the ED once again with superior vena cava syndrome. Soon after, due to compression, the patient developed a pericardial effusion with signs of heart failure and underwent mediastinal debulking. Surgical pathology revealed a $12.5 \mathrm{~cm}$ mature teratoma with extensive necrosis. He reported chronic fatigue, exertional dyspnea, and a racing heart without fever or chest pain and was placed on home oxygen and BiPAP due to chronic hypoxemia. A PET/ CT was performed to evaluate suspicions of GCT dissemination. Results showed abnormal hypermetabolic foci in the mediastinal lymph nodes, left hilum and subcarinal lymph nodes, periportal, peripancreatic and aortocaval lymph nodes. Hypermetabolic foci were also noted in multiple osseous lesions including the proximal femoral shaft, left trochanteric region, lumbar vertebra, and right third lateral rib. Subsequent left iliac bone core biopsy and FNA revealed bone marrow with numerous epithelioid granulomas in a background of normocellular bone marrow consistent with sarcoidosis. The patient never complained of bone pain.

\section{Discussion.}

The described patient was found to have multiple painless bone lesions on imaging studies related to his GCT. Given the extensive and well-documented history of GCT, the leading diagnosis after the PET/CT revealed the skeletal lesions was first thought to be metastatic disease. However it is important to keep in mind that at the time the patient already had an established diagnosis of Sarcoidosis. Other differential diagnosis for multiple skeletal lesions can include multiple myeloma, brown tumors of hyperparathyroidism, lymphoma, histiocytosis, osteomyelitis, Paget's disease, and fibrous dysplasia.

The incidence of Sarcoidosis with skeletal involvement has been speculated to range from $3 \%$ to $38 \% .^{5-7}$ However, skeletal Sarcoidosis may or may not cause significant musculoskeletal manifestations. Therefore, since most patients are asymptomatic and radiologic examination is not routinely performed, sarcoid bone lesions could go undetected and true incidence of skeletal Sarcoidosis could be underestimated. If it was not for our patient's GCT, he likely would not have required imaging studies.

Skeletal involvement has been documented more frequently in the phalanges as compared to the long bones, vertebra, skull and pelvic bones." Our patient had no involvement of the bones of his hands or feet but rather involved his long bones, pelvic bones, vertebra and ribs. Pure skeletal involvement is rare in the setting of Sarcoidosis. The presence of skeletal sarcoidosis does not seem to have an impact 
on the degree of respiratory dysfunction. ${ }^{8}$ However, it is difficult to assess to what degree Sarcoidosis contributed to his pulmonary symptoms as he had an extensive primary tumor located in his mediastinum.

Since our patient was being followed and treated for his GCT, we had the luxury of knowing that previous imaging studies did not show any skeletal involvement. It is quite certain that pulmonary sarcoidosis involvement preceded the skeletal involvement in this case. As stated above, since radiologic studies would not routinely be preformed, normally it would be extremely hard to determine whether skeletal sarcoidosis preceded pulmonary and mediastinal involvement or if sarcoidosis first presented with pulmonary and mediastinal manifestations.

Regarding laboratory testing, elevated inflammatory markers and ACE levels are more frequently found in sarcoidosis with skeletal involvement. ${ }^{9}$ However our patient had both pulmonary and skeletal involvement as well as a cancer, which could all contribute and affect inflammatory markers and alter lab data.

First line therapy for sarcoidosis has traditionally been systemic corticosteroids while refractory cases have often been managed with Methotrexate. ${ }^{10}$ The combination of history of GCT with prior surgery and chemotherapy, along with a wide spread dissemination of sarcoidosis in our patient, creates a challenge to the more traditional sarcoidosis treatment methods particularly because there is increased likelihood of infection due to impaired immunity.

In this case, the imaging features that were unexpectedly found to be sarcoid rather than metastasis would likely be considered as nonspecific and yield a broad differential diagnosis in many scenarios. Previous data suggests that, although rare, osseous involvement in sarcoidosis can be differentiated from a destructive lytic bone lesion by the absence of periostitis on conventional radiography. ${ }^{15}$ However, definitive diagnosis can only be made with biopsy, which is necessary to rule out metastatic disease. In the future, appropriate use of PET scan may help in timely detection of osseous involvement in patients with sarcoidosis. $^{16}$

\section{References.}

1. Iannuzzi MC, Rybicki BA, Teirstein AS. Sarcoidosis: review article. N Eng J Med 2007; 357:2153-65.

2. Pietinalho A, Hiraga $Y$, Hosoda $Y$, Lofroos AB, Yamaguchi M, Selroos 0 . The frequency of sarcoidosis in Finland and Hokkaido, Japan: a comparative epidemiological study. Sarcoidosis 1995; 12:61-7.

3. Milman N, Selroos O. Pulmonary sarcoidosis in the Nordic countries 1950 1982: epidemiology and clinical picture. Sarcoidosis 1990;7:50-7.

4. Sartoris DJ, Resnick D, Resnik C, Yaghmai I. Musculoskeletal manifestations of sarcoidosis. Sem Roentgenology 1985; 4:376-88.

5. Uehlinger E, Wurm K. Sarcoidosis of the skeleton: review of the literature and case report. ROFO Fortschr Geb Rontgenstr Nuklearmed 1976;125:11122.

6. Shorr AF, Murphy FT, Gilliland WR, Hnatiuk OW. Osseous disease in patients with pulmonary sarcoidosis and musculoskeletal Symptoms. Resp Medicine 2000; 94:228 -232.

7. Abril A, Cohen MD. Rheumatologic manifestations of sarcoidosis.Curr Opin Rheumatol 2004; 16(1):51.

8. Poyanli A, Poyanli 0 , Sencer $S$, et al. Vertebral sarcoidosis: imaging findings. Euro Radiol 2000; 10:92-4.

9. Beasley EW III, Peterman SB, Hertzler GL. An unusual form of tibial sarcoidosis. Am J Roentgenol 1987; 149:754-6.

10. Aberg C, Ponzo F, Raphael B, Amorosi E, Moran V, Kramer E. FDG Positron Emission Tomography of Bone Involvement in Sarcoidosis. Am J Roentgenol 2004; 182:975-7.

\section{Acknowledgements}

I would like to acknowledge Hipen Shah, a 4th Year Biology Major at New Jersey Institute of Technology and aspiring physician, for his help in completing the final steps of this project.

Conflict of Interest Statement a Funding

The Authors have no funding, financial relationships or conflicts of interest to disclose.

Cite as:

Rabinowitz E, Ogedegbe C, Feldman J. A Case of Sarcoidosis Disseminated to Skeletal Tissues. Int J Med Students 2013;1(1):43-5. 